\title{
Neurosurgery in a patient with Creutzfeldt-Jakob disease after pituitary derived growth hormone therapy in childhood
}

\author{
Sarah J Holmes, James W Ironside, Stephen M Shalet
}

\begin{abstract}
The case of a young man who had previously received pituitary derived growth hormone for treatment of radiation induced growth hormone deficiency is reported. He underwent neurosurgery for presumed recurrence of a posterior fossa tumour but was subsequently shown to have Creutzfeldt-Jakob disease, confirmed on necropsy. The risk of transmission of Creutzfeldt-Jakob disease by neurosurgical instruments is discussed. Since the occurrence of this case the Department of Health have issued guidelines concerning neurosurgery and ophthalmic surgery in patients who have previously received treatment with pituitary derived growth hormone and may therefore be at risk of developing Creutzfeldt-Jakob disease. Surgical instruments used on such patients should under no circumstances be reused, and should be destroyed after use.
\end{abstract}

(F Neurol Neurosurg Psychiatry 1996;60:333-335)

Keywords: Creutzfeldt-Jakob disease; neurosurgery; pituitary derived growth hormone

Creutzfeldt-Jakob disease was first reported in a recipient of cadaveric pituitary derived human growth hormone in $1985 . .^{12}$ Around 1900 children in the United Kingdom received cadaveric pituitary derived human growth hormone of the disease has been reported to range from four to 30 years in those patients who received pituitary derived growth hormone in the United Kingdom. ${ }^{2}$

We present a case of a young man who developed iatrogenic Creutzfeldt-Jakob disease after receiving pituitary derived growth hormone as a child. The case raises several important issues including the difficulty in distinguishing Creutzfeldt-Jakob disease from recurrent posterior fossa tumour in a patient with cerebellar signs, and the problems associated with neurosurgical intervention in such a patient.

\section{Case report}

The patient presented in 1971 , at the age of six years, with a six month history of intermittent headache and unsteadiness, and was found to have right sided cerebellar signs. $\mathrm{He}$ underwent a posterior fossa craniotomy which disclosed a large tumour in the right cerebellar hemisphere. The tumour was almost completely resected and was found to be an astrocytoma on pathological examination. $\mathrm{He}$ underwent radiotherapy to the whole head and spine ( $2700 \mathrm{cGy}$ delivered in 17 fractions over 22 days) with a booster dose to the posterior fossa ( $1000 \mathrm{cGy}$ delivered in four fractions over four days) and subsequently received a 16 month course of chemotherapy with vincristine.

Over the next few years he was noted to be growing poorly, and was shown to be growth hormone deficient in 1974 . He received treatment with pituitary derived growth hormone from 1975 to 1981 . He remained neurologically well during this time, and moved away from the area in 1982, at which time he was lost to follow up.

The next contact with the patient was in late January 1992, when his mother telephoned the hospital to report that he had developed symptoms of "shakiness" over the preceding four to six months. He was seen in the outpatient clinic in early February 1992, aged 26, when he was found to have right sided cerebellar signs comprising intention tremor, dysmetria, and nystagmus on rightward gaze. He also had dysarthria. Brain CT was reported as showing a mixed cystic and solid mass involving the right side of the cere\footnotetext{
before 1985, at which time it was withdrawn and replaced by recombinant human growth hormone, which is not associated with risk of transmission of Creutzfeldt-Jakob disease. There have now been 13 cases of CreutzfeldtJakob disease in the United Kingdom after childhood treatment with pituitary derived growth hormone. There is no diagnostic test for Creutzfeldt-Jakob disease, or for predicting the clinical onset of the disease in a person who may have been infected by previous exposure to pituitary derived growth hormone but who has not yet developed signs of the disease. Furthermore, there is wide variation in the latency period between infection and clinical onset of Creutzfeldt-Jakob disease; the time between the most recent exposure to pituitary

derived growth hormone and the clinical onset

\author{
Received 21 December 1994 \\ and in revised \\ Accepted 13 November 1995 \\ Professor S M Shalet \\ Endocrinology, Christi \\ Wilmslow Road, Manchester \\ Endocrinology,
Christie Hospital NHS
Trust, Wilmslow Road, \\ Manchester M20 4BX, \\ S M Shalet \\ Western General \\ Hospital, Edinburgh
}

Hospital NHS Trust,

EH4 2XU, UK
} 
Figure 1 The cerebellum shows pronounced neuronal loss in the granular and Purkinje cell layers, with reactive gliosis and numerous small vacuoles characteristic of spongiform change in the molecular layer (haematoxylin and eosin $\times 240$ ).
Figure 2 Immunocytochemistry for prion protein shows numerous densely staining plaque-like deposits within the granular layer, molecular layer, and white matter (avidinbiotin-peroxidase technique with haematoxylin counterstain $\times 240$ )

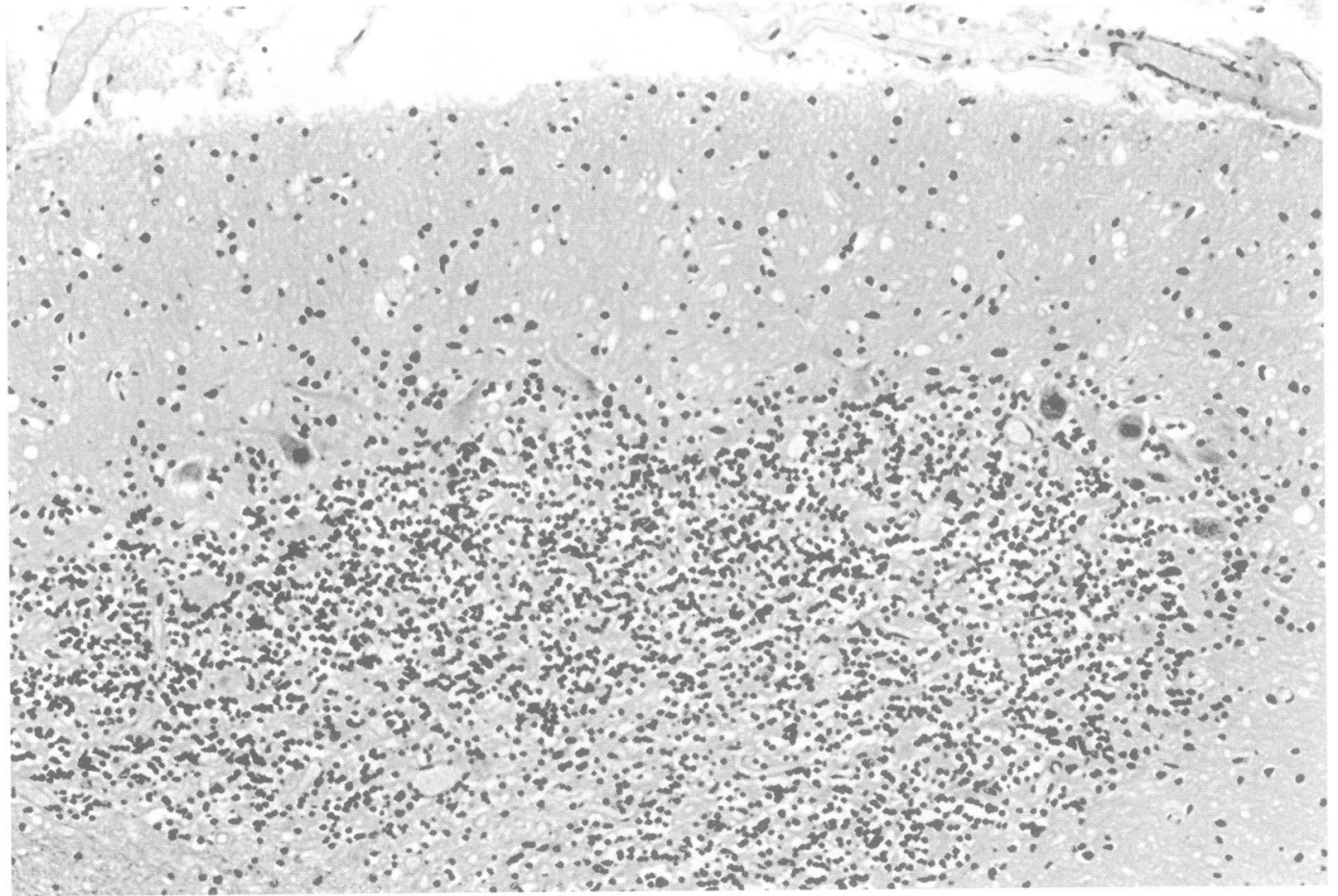

bellum. The appearance was reported as being compatible with a new or recurrent right sided posterior fossa tumour. The patient was subsequently referred to a neurosurgeon, and, in February 1992, he underwent a posterior fossa exploration. A cerebellar incision opened into the cyst, with apparent tumour mass visible on the anteriomedial wall. This mass was removed macroscopically. Pathological examination of the tissue removed at operation, however, showed no evidence of recurrent tumour, but a necrotising leucoencephalopathy consistent with radionecrosis.

The patient was well immediately after operation, but became confused five days later. Brain CT showed an increase in ventric- ular size. The patient underwent insertion of an external ventricular drain and subsequently required insertion of a ventriculoperitoneal shunt.

Postoperatively the patient continued to deteriorate steadily with increasing cerebellar ataxia and poor concentration and memory. He was living away from the area, and his family did not wish him to return for outpatient follow up, but in November 1992 the patient's father reported that the patient was hardly able to walk due to poor balance and required a wheelchair to mobilise. He was having frequent muscle twitching, especially involving the muscles of his upper limbs. At this time he was still just able to feed and dress himself,

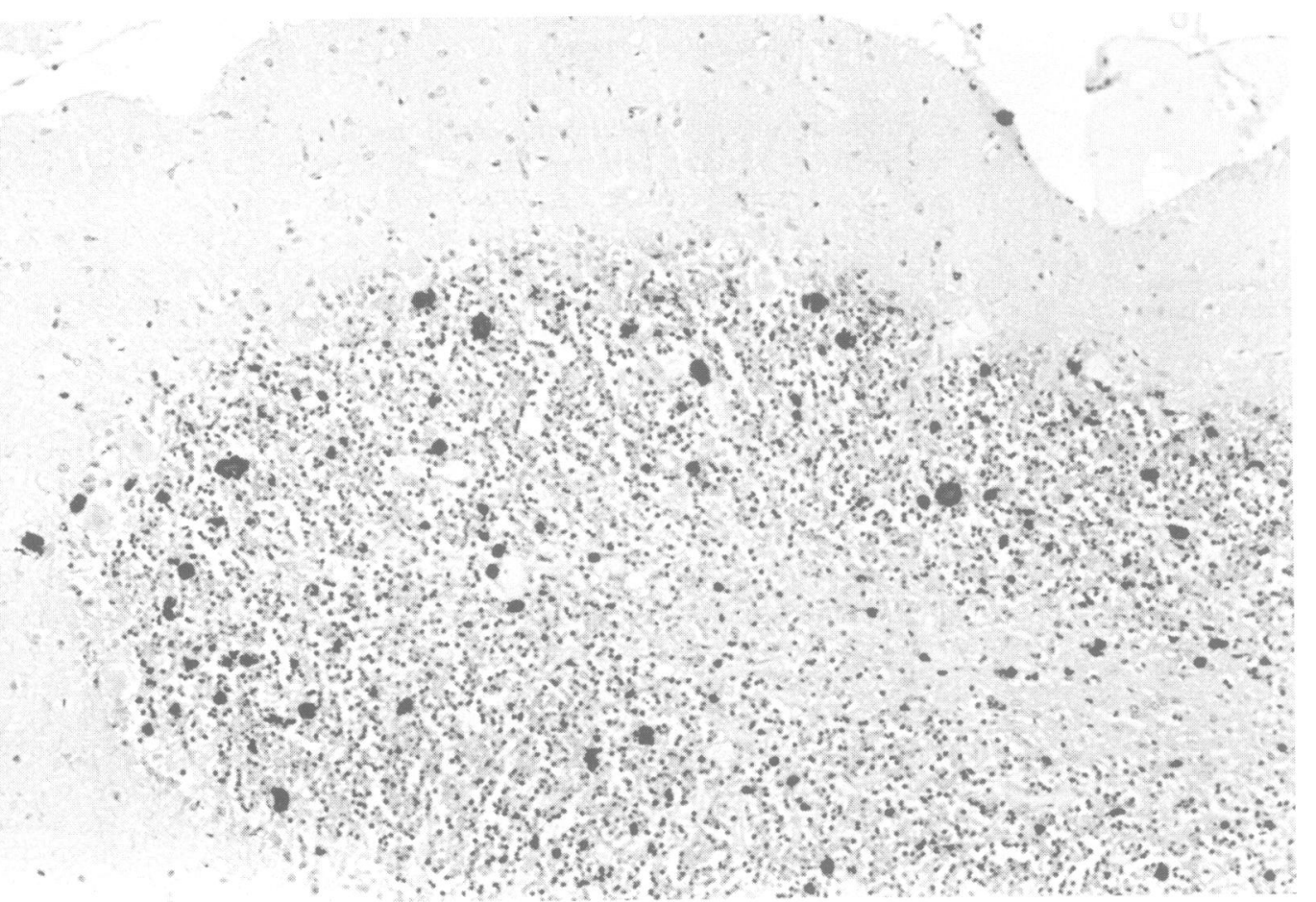


although his short term memory was now extremely poor, and his father described him as "dementing". By May 1993 he required feeding through a nasogastric tube. The patient died of bronchopneumonia in June 1993.

Necropsy examination of the brain showed gliosis, necrosis, and widespread degenerative changes in the white matter surrounding the cerebellar cystic lesion, similar to those reported in the previous biopsy specimen and consistent with necrotising leucoencephalopathy. Additional sections from the cerebellum and cerebral hemispheres showed the appearance of a spongiform encephalopathy, consistent with Creutzfeldt-Jakob disease (fig 1). Prion protein immunocytochemistry identified numerous plaque-like structures, particularly in the cerebellum and in a more sparse distribution in the cerebral cortex (fig 2). Prion protein immunostaining also showed a positive reaction in some areas of spongiform change, and in a perineuronal distribution in occasional fields within the cerebral cortex. These staining reactions are confirmatory of the diagnosis of Creutzfeldt-Jakob disease. There was no evidence of recurrent astrocytoma.

\section{Discussion}

Iatrogenic Creutzfeldt-Jakob disease was first reported in 1974 in a woman who had received a corneal transplant 18 months before the onset of symptoms of the disease. ${ }^{2}$ There are reports of transmission of CreutzfeldtJakob disease after stereotactic EEG depth recordings and after neurosurgery, suggesting contamination of neurosurgical instruments. ${ }^{2}$ Creutzfeldt-Jakob disease that seemed to have been acquired from a dura mater graft was first reported in 1987, and there have been several more such cases reported since. ${ }^{2}$ There are reported cases of Creutzfeldt-Jakob disease occurring in a neurosurgeon, a neuropathologist, two histopathology technicians, ${ }^{2}$ a physician who had previously trained as a pathologist, ${ }^{3}$ and a report of possible transmission to an orthopaedic surgeon by handling of dura mater, ${ }^{4}$ raising the possibility of transmission of Creutzfeldt-Jakob disease by handling infected tissue.

The incubation period of Creutzfeldt-Jakob disease in patients who were infected by neurosurgery has been reported as being 18 to 28 months, and 16 to 20 months in the two patients who acquired Creutzfeldt-Jakob disease by stereotactic electroencephalography, and is therefore considerably shorter than that in patients who acquire Creutzfeldt-Jakob disease by transmission from pituitary derived growth hormone. ${ }^{2}$ The presenting feature in patients with Creutzfeldt-Jakob disease transmitted by neurosurgery and by stereotactic encephalography is mental deterioration, unlike patients with Creutzfeldt-Jakob disease acquired from pituitary derived growth hormone, in whom the presenting feature is a cerebellar syndrome. ${ }^{12}$
Neuropathological studies of CreutzfeldtJakob disease occurring in recipients of pituitary derived growth hormone have shown that, by contrast with sporadic CreutzfeldtJakob disease, cerebellar involvement is particularly severe and often accompanied by the formation of prion protein amyloid plaques, as in this case. The severe cerebellar abnormalities correlate well with the main clinical features in such cases, which are also distinct from those in most cases of sporadic Creutzfeldt-Jakob disease, in which dementia and extrapyramidal symptoms are common presenting features and usually dominate the clinical picture. Molecular genetic analysis of the prion protein gene in pituitary derived growth hormone recipients who have developed Creutzfeldt-Jakob disease shows a significant predominance of patients $(92 \%)$ with allelic homozygosity at codon $129 .{ }^{5}$ The corresponding frequency in the normal population is around $50 \%$; it has been suggested that homozygosity at this locus may be a predisposing factor in the development of CreutzfeldtJakob disease in recipients of pituitary derived growth hormone.

The previously reported cases of transmission of Creutzfeldt-Jakob disease by neurosurgical instruments raise the possibility that other patients who underwent neurosurgery using the same instruments as were used in this patient could be at risk of developing Creutzfeldt-Jakob disease. Since this case occurred the Department of Health have issued guidelines concerning neurosurgery and ophthalmic surgery in patients with Creutzfeldt-Jakob disease, or in patients who may be at risk of developing the disease because of previous exposure to pituitary derived growth hormone. ${ }^{6}$ These guidelines were sent to all consultant neurosurgeons and advise that all instruments used at operation on such patients should under no circumstances be reused, and should be destroyed after use.

We are indebted to Dr VM Joglekar, consultant histopathologist, for access to pathological material from this patient. The gist, for access to pathological material from this patient. The
prion protein antibody (KG9) was kindly supplied by Dr CR prion protein antibody (KG9) was kindly supplied by Dr CR
Birkett, Compton. The CID Surveillance Unit is funded by the Birkett, Compton. The

1 Markus HS, Duchen LW, Parkin EM, et al CreutzfeldtJakob disease in recipients of human growth hormone in the United Kingdom: a clinical and radiographic study Qf Med 1992;297:43-51.

2 Brown P, Preece MA, Will RG. "Friendly fire" in medicine: hormones, homografts, and Creutzfeldt-Jakob disease. Lancet 1992;340:24-7.

3 Berger JR, David NJ. Creutzfeldt-Jakob disease in a physician: a review of the disorder in health care workers. Neurology 1993;43:205-6.

4 Weber T, Tumani H, Holdorff B, Collinge J, Palmer M, Kretzschmar HA, Felgenhauer K. Transmission of Lancet 1993;341:123-4.

5 Brown P, Cervenakova L, Goldfarb LG, et al. Iatrogenic Creutzfeldt-Jakob disease: an example of the interplay between ancient genes and modern medicine. Neurology between ancien

6 Wight A. PL(92)CO/4-Neuro and ophthalmic surgery procedures on patients with or suspected to have, or at procedures on patients with or suspected to have, or at
risk of developing, Creutzfeldt-Jakob disease (CID), or risk of developing, Creutzfeldt-Jakob disease (CJD), or
Gerstmann-Straussler-Scheinker syndrome (GSS). $f$ Public Health Med 1993;15:209-10. 